\title{
Custos e eficácia da toxina botulínica tipo A no tratamento do blefaroespasmo essencial e espasmo hemifacial
}

\author{
Costs and efficacy of type A botulinum toxin for the treatment of essential \\ blepharospasm and hemifacial spasm
}

\author{
Cintia Gomes Galvão Lasalvia ${ }^{1}$ \\ Luciano de Sousa Pereira ${ }^{2}$ \\ Marcos Carvalho da Cunha ${ }^{3}$ \\ Silvia Prado Smit Kitadai ${ }^{4}$
}

Trabalho realizado na Santa Casa de Misericórdia de São Paulo (SP) - Brasil, Departamento de Oftalmologia.

Médico voluntário da seção de óculo-plástica do Departamento de Oftalmologia da Faculdade de Ciências Médicas da Santa Casa de Misericórdia de São Paulo São Paulo (SP) - Brasil.

${ }^{2}$ Estagiário da seção de óculo-plástica do Departamento de Oftalmologia da Faculdade de Ciências Médicas da Santa Casa de Misericórdia de São Paulo - São Paulo (SP) - Brasil.

${ }^{3}$ Médico assistente da seção de óculo-plástica do Departamento de Oftalmologia da Faculdade de Ciências Médicas da Santa Casa de Misericórdia de São Paulo São Paulo (SP) - Brasil.

${ }^{4}$ Médica assistente do Departamento de Oftalmologia da Universidade de Santo Amaro - UNISA - São Paulo (SP) - Brasil.

Endereço para correspondência: Cintia Gomes Galvão Lasalvia. Rua Demóstenes, 1279 - São Paulo (SP) CEP 04614-015

E-mail: cintiagalvao@uol.com.br

Recebido para publicação em 27.07.2005

Última versão recebida em 24.01.2006

Aprovação em 30.01.2006

Nota Editorial: Depois de concluída a análise do artigo sob sigilo editorial e com a anuência da Dra. Silvana Artioli Schellini sobre a divulgação de seu nome como revisora, agradecemos sua participação neste processo.

\section{RESUMO}

Objetivo: Avaliar os custos do tratamento para blefaroespasmo essencial e espasmo hemifacial com toxina botulínica tipo A (Dysport $\left.{ }^{\circledR}\right)$, correlacionando-os com sua eficácia terapêutica. Métodos: Análise de 50 prontuários de pacientes com blefaroespasmo essencial e espasmo hemifacial, submetidos à terapia com Dysport ${ }^{\circledR}$, no período de abril de 2002 a maio de 2004 no setor de Óculo-Plástica da Santa Casa de São Paulo. Dos 50 pacientes, 27 apresentavam blefaroespasmo essencial e 23 espasmo hemifacial. Informações sobre grau de satisfação, queixas e custos pessoais foram obtidas mediante questionário. Os custos do medicamento e dos materiais foram pesquisados no almoxarifado e na farmácia da Santa Casa. Quanto ao custo das consultas, utilizou-se a tabela de pagamento do SUS. Para a estatística foram utilizados os testes de Wilcoxon e MannWhitney. Resultados: O custo total anual do tratamento foi de $\mathrm{R} \$ 1.239,32$ para o blefaroespasmo essencial e $\mathrm{R} \$ 661,72$ para o espasmo hemifacial. Para o paciente, o custo anual foi de $\mathrm{R} \$ 145,48$ para o blefaroespasmo essencial e $\mathrm{R} \$ 126,07$ para o espasmo hemifacial. Para o hospital, o custo anual foi de $\mathrm{R} \$ 1.095,84$ para o blefaroespasmo essencial e R\$ 535,65 para o espasmo hemifacial. O tratamento com Dysport ${ }^{\circledR}$ promoveu melhora funcional significativa nos dois grupos. Conclusão: O procedimento tem custo elevado, principalmente devido ao preço da toxina. Entretanto, pela análise econômica da saúde fica demonstrado que o procedimento possui excelente relação custo-benefício.

Descritores: Blefarospasmo/quimioterapia; Distonia; Espasmo hemifacial/quimioterapia; Toxina botulínica tipo A/uso terapêutico; Toxina botulínica tipo A/economia; Custos e análise de custo; Espasticidade muscular

\section{INTRODUÇÃO}

O blefaroespasmo essencial benigno (BEB) é uma doença idiopática que afeta o músculo orbicular oculi, observando-se como conseqüência o fechamento involuntário, espasmódico e bilateral das pálpebras ${ }^{(1-7)}$. Nas formas graves o paciente pode apresentar cegueira funcional ${ }^{(1,3,5)}$ e nas formas moderadas pode levar a uma limitação nas atividades diárias, como leitura, dificuldade para caminhar e trabalhar ${ }^{(1,3,7-9)}$.

O espasmo hemifacial (EH) caracteriza-se por contrações tônicas, paroxísticas e involuntárias, limitadas aos músculos de uma das hemifaces, inervados pelo sétimo nervo craniano. Geralmente tem início no músculo orbicular e se espalha progressivamente, comprometendo todos os músculos da hemiface acometida, persistindo durante o sono, ao contrário do que ocorre no $\mathrm{BEB}^{(1,8,10-14)}$. 
A toxina botulínica do tipo A (TBX-A) é uma neurotoxina que causa uma denervação química pelo bloqueio da liberação de acetilcolina na junção neuromuscular, aprovada pelo "Food and Drugs Administration" em 1989 para tratamento das distonias faciais e estrabismo. Em 1992 foi liberada para uso médico no Brasil, pelo Ministério da Saúde. O tratamento é ambulatorial, evitando-se o custo hospitalar de uso do centro cirúrgico ${ }^{(1,9,12)}$.

Os custos do tratamento do BEB e EH com TBX-A são muito pouco citados na literatura nacional e internacional. Trata-se de medicação importada, de custo elevado.

O custo é dividido em custos diretos, compostos pelos gastos com aquisição de materiais e medicamentos, e mão-deobra dos médicos oftalmologistas; e custos indiretos, como energia, água, utilização do ambulatório. Os custos diretos são assim denominados porque seus valores são de fácil identificação. Nos custos indiretos estão incluídos gastos que não possuem uma correta identificação de seus valores e quantidades $^{(15)}$.

O objetivo deste estudo é avaliar os custos do tratamento para BEB e EH com TBX-A, relacionando-o com sua eficácia.

\section{MÉTODOS}

Foram analisados 50 prontuários de pacientes com BEB e $\mathrm{EH}$, submetidos à terapia com TBX-A, no período de abril de 2002 a maio de 2004 no setor de Óculo-Plástica da Santa Casa de Misericórdia de São Paulo (SCMSP). Dos 50 pacientes, 27 apresentavam BEB e 23 EH. Foi aplicado o questionário complementar que indagava sobre grau de satisfação após a aplicação da TBX-A, custos pessoais e gastos com transporte, alimentação e perda de dia de trabalho.

O protocolo para preparação da droga do setor da ÓculoPlástica da SCMSP consiste em adicionar $5,0 \mathrm{ml}$ de soro fisiológico $0,9 \%$ sem conservante a um frasco de TBX-A, contendo 500 unidades internacionais (UI) $\left[\right.$ Dysport ${ }^{\circledR}$ Laboratório Porton Products, UK]. A solução foi, então, colocada em seringa de insulina e injetada, com agulha $12,7 \times 0,33 \mathrm{~mm}$, no subcutâneo em pontos pré-determinados da face ou hemiface acometidos, após limpeza da pele com álcool etílico. Dependendo da intensidade das contrações foram feitas aplicações em mais pontos. Cada ponto recebeu 10,0 UI e somente alguns pontos receberam 20,0 UI de toxina. Um mesmo frasco de Dysport foi utilizado em média para 4-5 pacientes. O procedimento foi realizado no próprio ambulatório.

Os pacientes foram avaliados 15, 30 e 60 dias, após a aplicação, ficando o próximo retorno a critério da avaliação clínica do espasmo. No retorno de 30 dias foi feita avaliação quantitativa do espasmo e prejuízo funcional aplicando-se a escala Baylor University Rating Scale (BURS), que consiste na seguinte escala: zero - assintomático; 1- distonia leve, sem prejuízo funcional; 2- distonia moderada, com déficit funcional mínimo; 3- distonia moderada, com comprometimento funcional mode- rado; 4- distonia intensa e incapacitante comparando-se com a avaliação prévia ao tratamento ${ }^{(9)}$. Neste mesmo retorno, o paciente pôde responder sobre o grau de satisfação ao tratamento que foi classificado em: melhora total $(100 \%)$; acentuada $(>50 \%)$; discreta $(<50 \%)$; sem melhora $(0 \%)$. Sendo assim, o paciente e o médico puderam classificar o grau de satisfação.

Para o cálculo com os gastos do paciente e acompanhantes com transporte usamos as tarifas de transporte por ônibus, metrô e trem metropolitano do município de São Paulo, para um paciente e um acompanhante poderem comparecer às consultas durante um ano de tratamento.

Foi realizado o levantamento dos materiais e medicamentos utilizados no procedimento de aplicação da TBX-A e seus custos. Os preços foram pesquisados diretamente nos departamentos responsáveis pela compra e estoque de materiais e medicamentos da SCMSP (almoxarifado e farmácia). Quanto ao custo das consultas, utilizou-se a tabela de pagamento pelo Sistema Único de Saúde (SUS). Os custos dos honorários dos médicos envolvidos neste procedimento não foram considerados, pois estes estão em trabalho voluntário. Também foi feito levantamento de custos com alimentação e perda do dia de trabalho durante o período da consulta, por meio de questionários.

A análise de todos os custos está baseada nos valores em reais pesquisados no mês de junho de 2004 .

O Comitê de Ética da Universidade Federal de São Paulo (UNIFESP) deu parecer favorável sobre este estudo (No 1018/ 04) e os pacientes consentiram com o tratamento proposto.

Para a comparação entre a classificação funcional antes e depois do tratamento entre os grupos BEB e EH foi utilizado o teste de Mann-Whitney. Para a comparação dentro dos grupos BEB e EH foi dutilizado o teste de Wilcoxon. Convencionou-se como risco $\alpha \leq 0,05$ ou $5 \%$. Os valores significativos estão assinalados com asterisco $(*)^{(16)}$.

\section{RESULTADOS}

As características da população estudada com BEB e EH encontram-se resumidas no quadro 1.

Para o cálculo de custos com transportes, foi estabelecida uma média para cada tipo de transporte usado: ônibus, metrô e trem metropolitano. Analisando o protocolo de retornos deste Serviço foi constatado que durante um ano são estabelecidos oito retornos para $\mathrm{BEB}$ e seis para $\mathrm{EH}$, que multiplicados por duas pessoas perfazem 16 e 12 passagens. Considerando que há ida e volta resultam 32 e 24 passagens para BEB e EH, respectivamente. $\mathrm{O}$ resultado da média total de gastos com transportes em um ano foi de $\mathrm{R} \$ 58,56$ para BEB e $\mathrm{R} \$ 44,00$ para $\mathrm{EH}$.

Os custos de alimentação para o paciente e acompanhante em razão da consulta foram, em média, de $\mathrm{R} \$ 2,00$ por consulta. Considerando que houveram oito retornos anuais para o grupo BEB os custos totalizam $\mathrm{R} \$ 16,00$ por ano e para o grupo $\mathrm{EH}$, com seis retornos, $\mathrm{R} \$ 12,00$.

Nos cálculos referentes à perda do dia de trabalho para 
comparecimento à consulta, com base nos questionários, somente alguns pacientes de cada grupo tiveram desconto do dia de trabalho, ou mesmo deixaram de ganhar por não trabalhar. Foi então calculada a média por grupo por consulta e anual, ficando em $\mathrm{R} \$ 16,29$ por consulta para BEB e $\mathrm{R} \$ 28,69$ para $\mathrm{EH}$. Considerando que os pacientes do grupo BEB retornam ao ambulatório oito vezes ao ano e o grupo EH retornam seis vezes ao ano, totaliza $\mathrm{R} \$ 70,82$ para BEB e $\mathrm{R} \$ 70,07$ para EH.

Os materiais e medicamentos usados para o procedimento da aplicação da TBX-A são: TBX-A (Dysport $\left.{ }^{\circledR}\right)$, seringa, agulha, álcool, gaze, soro fisiológico e luva de procedimento. Os preços foram pesquisados na farmácia e almoxarifado e são $\mathrm{R} \$ 830,00 ; \mathrm{R} \$ 0,17 ; \mathrm{R} \$ 0,09 ; \mathrm{R} \$ 0,80 ; \mathrm{R} \$ 0,32$ e $\mathrm{R} \$ 0,19$, respectivamente. Os cálculos foram feitos para uma aplicação para ambos os grupos e feito também o cálculo anual.

$\mathrm{O}$ grupo BEB faz em média quatro aplicações anuais e o grupo EH três aplicações anuais. Sendo assim, o custo anual com materiais para o grupo BEB é de $\mathrm{R} \$ 4,16$ e $\mathrm{R} \$ 2,28$ para EH. Já o custo anual com medicamentos ficou em $\mathrm{R} \$ 1.029,28 \mathrm{e}$ $\mathrm{R} \$ 488,07$, respectivamente.
O custo por consulta para o SUS foi de R $\$ 7,55$. Sabendose que o grupo dos pacientes com BEB retorna oito vezes por ano ao ambulatório e o grupo de pacientes com EH seis vezes ao ano, totaliza $\mathrm{R} \$ 60,40$ e $\mathrm{R} \$ 45,30$ respectivamente.

$\mathrm{Na}$ tabela 2 mostramos os pacientes quanto à melhora funcional classificada na Escala de Baylor University Rating Scale (BURS).

Na tabela 3 vemos o resultado quanto ao grau de satisfação relatado pelo paciente.

O grupo BEB faz em média quatro aplicações de TBX-A ao ano e o grupo EH faz em média três aplicações ao ano. O custo total anual do tratamento é de $\mathrm{R} \$ 1.239,32$ para o BEB e $\mathrm{R} \$ 661,72$ para o EH. Para o paciente, o custo anual é de $\mathrm{R} \$ 145,48$ para o BEB e R \$ 126,07 para o EH e para o Hospital, é de $\mathrm{R} \$ 1.095,84$ para o BEB e R $\$ 535,65$ para o EH.

\section{DISCUSS ÃO}

Os custos do transporte representam $\mathrm{R} \$ 58,56$ para BEB e $\mathrm{R} \$ 44,00$ para EH. Optou-se por apresentar os custos de loco-

\begin{tabular}{|lccccc|}
\hline \multicolumn{4}{|l}{ Quadro 1. Característica da população portadora de blefaroespasmo essencial benigno e espasmo hemifacial tratada com TBX-A na Santa } \\
Casa de Misericórdia de São Paulo
\end{tabular}

\begin{tabular}{|c|c|c|c|}
\hline & Custo por unidade & Custo por aplicação para BEB & Custo por aplicação para EH \\
\hline TBX-A (Dysport $\left.{ }^{\circledR}\right)$ & $\mathrm{R} \$ 830,00$ & $\mathrm{R} \$ 257,30^{\mathrm{a}}$ & $\mathrm{R} \$ 162,68^{\mathrm{c}}$ \\
\hline SF $0,9 \%(10 \mathrm{ml})$ & $\mathrm{R} \$ \quad 0,19$ & $\mathrm{R} \$ 0,029^{\mathrm{b}}$ & $\mathrm{R} \$ 0,018^{\mathrm{d}}$ \\
\hline Total & $\mathrm{R} \$ 830,19$ & $\mathrm{R} \$ 257,32$ & $\mathrm{R} \$ 162,69$ \\
\hline \multicolumn{4}{|c|}{$\begin{array}{l}\text { BEB= blefaroespasmo essencial benigno; } E H=\text { espasmo hemifacial; TBX-A= toxina botulínica } A ; S F=\text { soro fisiológico } \\
\text { a = por aplicação é utilizada média de } 155 \mathrm{UI}=\mathrm{R} \$ 257,30 \text {, sabendo-se que um frasco de } 500 \mathrm{UI}=\mathrm{R} \$ 830,00 ; \mathrm{b}=\text { por aplicação é utilizada média de } 1,55 \mathrm{ml}= \\
\mathrm{R} \$ 0,029 \text {, sabendo-se que uma ampola de } 10 \mathrm{ml}=\mathrm{R} \$ 0,19 ;{ }^{\circ}=\text { por aplicação é utilizada média de } 98 \mathrm{UI}=\mathrm{R} \$ 162,68 \text {, sabendo-se que um frasco de } 500 \mathrm{UI}=\mathrm{R} \$ 830,00 \text {; } \\
\text { d= por aplicação é utilizada média de } 0,98 \mathrm{ml}=\mathrm{R} \$ 0,018 \text {, sabendo-se que uma ampola de } 10 \mathrm{ml}=\mathrm{R} \$ 0,19 \text {. }\end{array}$} \\
\hline
\end{tabular}

\begin{tabular}{|c|c|c|c|c|c|c|c|c|c|c|}
\hline \multirow[b]{2}{*}{ Grau } & \multicolumn{5}{|c|}{ Antes do tratamento } & \multicolumn{5}{|c|}{1 mês após o tratamento } \\
\hline & 4 & 3 & 2 & 1 & 0 & 4 & 3 & 2 & 1 & 0 \\
\hline $\mathrm{EH}$ ( $\mathrm{n}^{\circ}$ de pacientes) & 4 & 18 & 1 & 0 & 0 & 0 & 0 & 0 & 12 & 11 \\
\hline \multicolumn{11}{|c|}{$\begin{array}{l}\text { BEB= blefaroespasmo essencial benigno; EH= espasmo hemifacial; TBX-A= toxina botulínica } \mathrm{A} ; \mathrm{n}^{\circ}=\text { número. } \\
\text { Classificação de BURS(9): Grau } 0=\text { assintomático; Grau 1= distonia leve, sem prejuízo funcional; Grau 2= distonia moderada, com déficit funcional mínimo; Grau } 3= \\
\text { distonia moderada, com comprometimento funcional moderado; Grau } 4=\text { distonia intensa e incapacitante }\end{array}$} \\
\hline
\end{tabular}




\begin{tabular}{|c|c|c|c|c|}
\hline Grau de melhoria & $100 \%$ & $>50 \%$ & $<50 \%$ & $0 \%$ \\
\hline BEB & $48,14 \%$ & $44,44 \%$ & $8,69 \%$ & - \\
\hline $\mathrm{EH}$ & $47,82 \%$ & $52,17 \%$ & - & - \\
\hline
\end{tabular}

\begin{tabular}{|c|c|c|}
\hline & BEB & EH \\
\hline Transporte & $\mathrm{R} \$ 58,66$ & $\mathrm{R} \$ 44,00$ \\
\hline Alimentação & $\mathrm{R} \$ 16,00$ & $\mathrm{R} \$ 12,00$ \\
\hline Perda do dia de trabalho & $\mathrm{R} \$ 70,82$ & $\mathrm{R} \$ 70,07$ \\
\hline Materiais & $\mathrm{R} \$ \quad 4,16$ & $R \$ 2,28$ \\
\hline Medicamentos & $\mathrm{R} \$ 1.029,28$ & $\mathrm{R} \$ 488,07$ \\
\hline Consultas & $R \$ 60,40$ & $\mathrm{R} \$ 45,30$ \\
\hline Total & $R \$ 1.239,32$ & $\mathrm{R} \$ 661,72$ \\
\hline
\end{tabular}

moção apenas dentro da cidade, pois os pacientes atendidos moravam na cidade de São Paulo. Os custos para alimentação no período da consulta, que foram $\mathrm{R} \$ 16,00$ para o BEB e $\mathrm{R} \$ 12,00$ para o $\mathrm{EH}$. Isto ocorre provavelmente porque os pacientes passam longas horas fora de casa. Os custos referentes à perda do dia de trabalho em razão da consulta que foram $\mathrm{R} \$ 70,82$ para o BEB e R \$70,07 para o EH. Muitos pacientes já são aposentados, porém, os que trabalham por conta própria não recebem, e os que são assalariados são descontados no dia em que se ausentam do trabalho para o tratamento.

A tabela 1, que analisa os gastos com materiais e medicamentos, mostra que os custos são maiores para os pacientes com BEB. Isso se deve provavelmente à duração do efeito da TXB-A nesses pacientes (cerca de três meses). Nos pacientes portadores de $\mathrm{EH}$ o efeito persiste por quatro a seis meses ${ }^{(1)}$.

A análise de custo-benefício é um instrumento de planejamento e avaliação de serviços em saúde. Para uma análise de acordo com a perspectiva esperada pelos familiares, os gastos referentes à aquisição de bens (medicamentos, colírios) e serviços (transporte, alimentação, consultas e procedimentos) podem ser considerados como custos, porque representam gastos para a produção de outro bem ou serviço como a visão, a melhora do espasmo, a possibilidade de convívio social e de novamente ter atividade normal ${ }^{(18-19)}$. Levando-se em conta a análise econômica da saúde que avalia o custo-benefício para o paciente apresentamos na tabela 2 e 3 a avaliação da melhora funcional e grau de satisfação do paciente em relação ao tratamento.

Neste estudo o consumo de material e medicamento representa custo direto para a SCMSP, e o custo maior é referente a TBX-A $\left(\right.$ Dysport $\left.^{\circledR}\right)$.
A melhora dos sintomas do BEB e do EH é evidente após o tratamento com a TBX-A. Na tabela 3, nota-se um elevado grau de satisfação dos pacientes após um mês de tratamento. A aplicação ambulatorial da TBX-A é um tratamento eficaz no controle temporário do $\mathrm{BEB}$ e EH, e uma boa alternativa ao tratamento cirúrgico.

\section{CONCLUSÕES}

A análise de custos do tratamento para o BEB e EH com TBX-A demonstrou que o procedimento tem gasto elevado, mas possui excelente custo-benefício. O tratamento com a TBX-A promove melhora funcional significante do espasmo nos pacientes com BEB e EH. São necessários mais estudos para melhor relacionar dose de toxina, tempo de duração de efeito, incidência de efeitos colaterais e, principalmente, custos, pois se trata de uma doença crônica, incapacitante, para a qual a melhor opção terapêutica, no momento, é a TBX- A.

\section{ABSTRACT}

Purpose: To evaluate the costs and efficacy of type A botulinum toxin in the treatment of essential blepharospasm and hemifacial spasm. Methods: Pacients with essential blepharospasm and hemifacial spasm had their files analyzed. All patients were treated with type A botulinum toxin (Dysport ${ }^{\circledR}$ ) between April 2002 and May 2004 at the Oculoplastic Clinics of "Santa Casa de São Paulo". Twenty-seven patients presented essential blepharospasm and 23 presented hemifacial spasm. Information about the patient's degree of satisfaction after treatment, complaints and personal costs were recorded by a questionnaire, and information about the costs of Dysport ${ }^{\circledast}$ treatment were obtained at the administration department of "Santa Casa de São Paulo". Wilcoxon and MannWhitney tests were used for statistical analysis. Results: 1The annual treatment costs were $\mathrm{R} \$ 1,239.32$ for essential blepharospasm and $\mathrm{R} \$ 661.72$ for hemifacial spasm. 2- The patient's annual costs were $\mathrm{R} \$ 145.48$ for essential blepharospasm and R\$ 6.07 for hemifacial spasm. 3- The hospital's annual costs for the treatment were $\mathrm{R} \$ 1,095.84$ for essential blepharospasm and $\mathrm{R} \$ 535.65$ for hemifacial spasm. 4- Dysport $^{\circledR}$ treatment is successful in both essential blepharospasm and hemifacial spasm. Conclusions:. The costs of essential blepharospasm and hemifacial spasm treatment with Dysport ${ }^{\oplus}$ are high, mainly because of the toxin price. On economic analysis of health, we can conclude that this procedure has an excellent cost-benefit ratio.

Keywords: Blepharospasm/drug therapy; Dystonia; Hemifacial spasm/drug therapy; Botulinum toxin type A/therapeutic use; Botulinum toxin type A/economics; Costs and cost analysis; Muscle spasticity 


\section{REFERÊNCIAS}

1. Osaki MH, Belfort Jr R. Qualidade de vida e custos diretos em pacientes com blefaroespasmo essencial e espasmo hemifacial, tratados com toxina botulínica A. Arq Bras Oftalmol. 2004;67(1):43-9.

2. Demer JD, Holds JB, Hovis LA. Ocular movements in essential blepharospasm. Am J Ophtalmol. 1990;110(6):674-82.

3. Kennedy RH, Waller RR, Henderson JW, Kesty KR. Management of blepharospasm. Ophthal Plast Reconstr Surg. 1985;1(4):253-61.

4. Defazio G, Livrea P. Epidemiology of primary blepharospasm. Mov Disord. 2002;17(1):7-12.

5. Lew MF. Review of the FDA-approved uses of botulinum toxins, including data suggesting efficacy in pain reduction. Clin J Pain. 2002;18(6 Suppl):S142-6.

6. Kowal L, Davies R, Kiely PM. Facial muscle spasms: an Australian study. Aust N Z J Ophthalmol. 1998;26(2):123-8.

7. Lindeboom R, De Haan R, Aramideh M, Speelman JD. The blepharospasm disability scale: an instrument for the assessment of functional health in blepharospasm. Mov Disord. 1995;10(4):444-9.

8. Shorr N, Seiff SR, Kopelman J. The use of botulinum toxin in blepharospasm. Am J Ophthalmol. 1985;99(5):542-6.
9. Lucci LMD. Blefaroespasmo essencial benigno. Arq Bras Oftalmol. 2002;65(5): 585-9.

10. Barbosa ER, Silva HC, Haddad MS, Bittar MS. Blefaroespasmo: tratamento com toxina botulínica. Rev Hosp Clin Fac Med S Paulo. 1996;51(6):220-3.

11. Anderson RL, Patel BC, Holds JB, Jordan DR. Related Articles, Links Blepharospasm: past, present, and future. Ophthal Plast Reconstr Surg. 1998;14(5): 305-17.

12. Cunha MC, Aguire OP, Souza-Dias CR. Tratamento do espasmo facial unilateral com toxina botulínica tipo A. Arq Bras Oftalmol. 1998;61(1):54-60.

13. Barbosa ER, Costa MDL, Staut CC, Bacheschi LA, Bittar MS. Espasmo hemifacial familiar: relato de dois casos. Arq Neuropsiquiatr. 1998;56(1):111-5.

14. Schellini SA, Matai O, Igami TZ, Padovani CR, Padovani CP. Essential blepharospasm and hemifacial spasm: characteristic of the patient, botulinum toxin A treatment and literature review. Arq Bras Oftalmol. 2006;69(1):23-6.

15. Martins D. Gestão financeira de Hospitais. $2^{a}$ ed. São Paulo: Saraiva; 2001.

16. Siegel S, Castellan NJ Jr. Non parametric statistics. 2nd ed. New York: McGraw-Hill; 1988.

17. Andrade LA, Borges V, Ferraz HB, Azevedo-Silva SM. Experiência com aplicação de toxina botulínica A em 115 pacientes. Arq Neuropsiquiatr. 1997;55(3B):553-7.

18. Martins D. Contabilidade de custos. 4a ed. São Paulo: Atlas; 1990.

19. Martins D. Custos e orçamentos hospitalares. São Paulo: Saraiva; 2001. 